\title{
ANÁLISIS PRELIMINAR DE LAS PROPIEDADES PSICOMÉTRICAS DE UN INSTRUMENTO PARA EVALUAR LAS ACTITUDES HACIA LA SEXUALIDAD DE LAS PERSONAS CON DISCAPACIDAD FÍSICA (ASEX-PDF)'
}

\author{
Olga Fernández-García \\ Dpto de Psicología Evolutiva y de la Educación \\ Universitat de València \\ Olga.Fernandez-Garcia@uv.es \\ Verónica Estruch-García \\ 2Dpto de Psicología Evolutiva y de la Educación \\ Universitat de València \\ María Dolores Gil-Llario \\ Dpto de Psicología Evolutiva y de la Educación \\ Universitat de València
}

Recepción Artículo: 13 mayo 2021 Admisión Evaluación: 13 mayo 2021 Informe Evaluador 1: 26 mayo 2021 Informe Evaluador 2: 28 mayo 2021 Aprobación Publicación: 01 junio 2021

\section{RESUMEN}

La salud sexual de las personas con discapacidad física (PDF) no sólo se ve influenciada por las limitaciones derivadas de la propia discapacidad sobre todo a la hora de iniciar contactos sino también, y sobre todo, por la estigmatización social. Creencias como que poseer un cuerpo con una funcionalidad no normativa puede interferir en la conducta sexual y en su función reproductiva son muestras de ello. Así mismo, se ha comprobado que la familia y los profesionales que se proporcionan apoyos a las PDF son el principal agente de formación, por lo que sus actitudes hacia la sexualidad de este colectivo van a condicionar su desarrollo sexual y la vivencia que hagan de la misma. Dada la importancia de estas actitudes y la escasez de instrumentos fiables dirigidos a evaluarlas, el objetivo de este trabajo ha sido construir y validar un instrumento de evaluación de las actitudes hacia la sexualidad de las personas con discapacidad física (ASEX-PDF). Para ello, se contó con 554 participantes ( $41.3 \%$ hombres y $58.7 \%$ mujeres) con edades comprendidas entre 20 y 92 años, de los cuales 100 eran profesionales que trabajan con este colectivo y 454 pertenecían a la población general. El Análisis Factorial Exploratorio extrajo una estructura unifactorial, que fue verificada y corregida a través del Análisis Factorial Confirmatorio, mostrando unos buenos índices de bondad de ajuste. La consistencia interna de la escala total fue de .79 ( de Cronbach) y de .89 ( ordinal). Así mismo, las correlaciones con una escala afín resultaron significativas y en la dirección esperada. En conclusión, la ASEX-PDF se presenta como un instrumento válido y fiable que puede resultar útil en la exploración de las actitudes hacia la sexualidad de las PDF.

Palabras clave: instrumento; actitudes; sexualidad; discapacidad física; propiedades psicométricas 


\title{
ANÁLISIS PRELIMINAR DE LAS PROPIEDADES PSICOMÉTRICAS DE UN INSTRUMENTTO PARA EVALUAR LAS ACTITUDES HACIA LA SEXUALIDAD DE LAS PERSONAS CON DISCAPACIDAD FÍSICA (ASEX-PDF)
}

\begin{abstract}
Preliminary analysis of the psychometric properties of an instrument to assess attitudes towards sexuality of people with physical disabilities (asex-pdf). People with physical disabilities (PPD) do not necessarily have to be less sexually active than people without disabilities. However, they experience poorer sexual health due, on the one hand, to the frequent belief that not having a body with a normative functionality may interfere with their sexual behaviour and reproductive function, and, on the other hand, to the rejection and/or denial of their sexuality that they perceive from their more or less close environment. Likewise, it has been proven that the family and the professionals who are in contact with the PPD are the main agents of training, so that their attitudes towards sexuality in this group will condition their sexual development and the experience they have of it. Therefore, the aim of this work has been to construct and validate an instrument to assess attitudes towards sexuality of people with physical disabilities (ASEX-PDF) in the Spanish context. There were 554 participants (41.3\% men and 58.7\% women) aged between 20 and 92 years, of whom 100 were professionals in contact with this group and 454 belonged to the general population. The Exploratory Factor Analysis (EFA) yielded a onefactor structure, which was verified and corrected through the Confirmatory Factor Analysis (CFA), showing good goodness-of-fit indices. The internal consistency of the total scale was .79 (Cronbach's ) and .89 (ordinal $\Omega$ ). Correlations with a related scale were also significant and in the expected direction. In conclusion, the ASEX-PDF is presented as a valid and reliable instrument that can be useful in exploring attitudes towards sexuality in PPD.
\end{abstract}

Keywords: instrument; attitudes; sexuality; physical disability; psychometric properties

\section{INTRODUCCIÓN}

Las personas con discapacidad tradicionalmente han constituido uno de los grupos más marginados, al presentar resultados académicos inferiores, peor atención en los servicios sanitarios, menor participación en la economía y tasas de pobreza más altas que las personas sin discapacidad (Martínez-Ríos, 2013; WHO y World Bank, 2011). En el caso de las personas con discapacidad física (PDF), se ha documentado que las enfermedades que frecuentemente padecen, el aislamiento social al que se ven expuestas y, sobre todo, los problemas sexuales que deben afrontar, contribuyen a explicar la baja satisfacción vital que presentan (Salehi, et al., 2015), que va acompañada de problemas de autoestima, frustración y soledad (Torices Rodarte, 2007).

Las PDF pueden tener una actividad sexual semejante a la de las personas sin discapacidad, pero experimentan peor salud sexual (Jemtå, Fugl-Meyer y Öberg, 2008). Las investigaciones constatan que las PDF tienen información limitada sobre sexualidad, reproducción y relaciones afectivas, lo que repercute directamente en sus experiencias, confianza y habilidades para establecer relaciones sexuales seguras y para denunciar situaciones de abuso 0 superioridad sexual (East y Orchard, 2014). Así mismo, pueden presentar con mayor frecuencia problemas en el funcionamiento sexual y son más dependientes de los demás, lo que resulta un hándicap a la hora de encontrar pareja afectiva y/o sexual, ya que las personas sin discapacidad alegan que sería incomodo socialmente y que no estarían satisfechas sexualmente si tuvieran una pareja con discapacidad física (Esmail et al., 2010; Marini et al., 2011). Esto evidencia que, aunque algunos estudios reportan que la población general muestra una buena aceptación, tolerancia y conciencia hacia estos aspectos de la vida de las PDF (Chou, Lu, y Lin, 2018; Morell-Mengual et al., 2017; Caballero-Gascón et al., 2017), las actitudes estigmatizadas hacia la expresión de la sexualidad de este colectivo aún están presentes. Creen que no poseer un cuerpo con una funcionalidad normativa puede interferir en su conducta sexual y su función reproductora, prefiriendo por ello no intimar con PDF (Torices Rodarte, 2007).

Así mismo, estas actitudes negativas se extienden también entre las personas del entorno más cercano de las PDF, como son los padres o familiares y los profesionales que están en contacto con ellos, quienes tradicionalmente han manifestado miedo, rechazo y/o negación hacia la sexualidad de este colectivo (Torices Rodarte, 2007). Ambos constituyen los principales agentes de formación de las PDF por lo que deben acompañarles, de manera afectiva, en el descubrimiento de su sexualidad y proporcionarles información útil que resuelva sus inquietudes (Valvano et al., 2014). En este sentido, cabe matizar que, el personal que trabaja a diario con ellos 
parece mostrarse mucho más favorable respecto a la promoción de un desarrollo sexual normativo en las PDF que los propios padres (Ride y Newton, 2018). Ejemplo de ello es el surgimiento de la figura del asistente 0 acompañante sexual, quien tras realizar una completa formación, se profesionaliza en el acompañamiento sexual, afectivo y erótico de hombres y mujeres con discapacidad.

Sin embargo, aunque existen evidencias como la expuesta anteriormente de que se está produciendo un cambio general en el paradigma actitudinal, son escasos los estudios que se hacen eco de estos progresos, debido, en parte, a la falta de instrumentos para evaluar las actitudes hacia la sexualidad de las PDF que hayan demostrado su eficacia previamente. Los estudios que han abordado este asunto, en la mayoría de los casos, han elaborado un instrumento ad hoc para dicha investigación (Hasson-Ohayon, et al. 2014) que posteriormente no han validado, han empleado escalas centradas en la evaluación de las actitudes hacia la sexualidad de las personas con discapacidad intelectual (PDI) y no física (Zárate y Calderón, 2010), y/o han usado escalas que evalúan las actitudes hacia diferentes aspectos de las personas con discapacidad (personalidad, ocupación, sociabilidad, etc.) que incluyen únicamente algún ítem sobre sexualidad (García Ortuño, 2017; Kravetz et al., 1994).

Así, teniendo en cuenta la repercusión que las actitudes de la población general y, sobre todo, de las personas cercanas a las PDF tienen sobre este colectivo, y ante las limitaciones metodológicas existentes para evaluar específicamente las actitudes hacia la sexualidad de las PDF, el presente estudio tiene el propósito de crear y analizar las propiedades psicométricas de un instrumento elaborado para evaluar las actitudes hacia la sexualidad de las PDF en el contexto español.

\section{MÉTODO}

\section{Participantes}

Un total de 554 personas participaron en este estudio transversal, de los cuales el 18.1\% eran profesionales que trabajaban con PDF y el 81.9\% eran personas de la población general.

La muestra se compone de un $41.3 \%$ de hombres y un $58.7 \%$ de mujeres, con una representación mayoritaria de personas que se identifican como heterosexuales (91.1\%), frente al $3.3 \%$ que afirmaron ser homosexuales y otro $5.6 \%$ bisexuales. La distribución por edad se dividió en un $55.6 \%$ de participantes con edades comprendidas entre 20 y 35 años, un $27.8 \%$ de entre 36 y 51 años, un 15.7\% que tenían entre 52 y 66 años y un $0.9 \%$ con edades de 67 a 92 años. Respecto al nivel de estudios, la mayoría de la muestra tenía estudios superiores (50.7\%), mientras el 31.6\% tenía estudios de secundaria, el 15.5\% el graduado escolar y una minoría no tenía estudios (2.2\%). En la Tabla 1 se muestran los porcentajes en cuanto al sexo, edad y nivel de estudios de los dos grupos que participaron en el estudio: población general y profesionales.

Tabla 1 Descripción de los participantes

Población general (\%) Profesionales (\%)

\begin{tabular}{lcc}
\hline Género & 39.43 & 50.00 \\
Hombres & 60.57 & 50.00 \\
$\quad$ Mujeres & & \\
Edad & 63.44 & 20.00 \\
20-35 años & 21.37 & 57.00 \\
36-51 años & 14.32 & 22.00 \\
52-66 años & 0.88 & 1.00 \\
67-92 años & & \\
Estudios & 2.64 & 0.00 \\
$\quad$ Sin estudios & 18.72 & 1.00 \\
Graduado escolar & 24.67 & 63.00 \\
Estudios de secundaria & 53.96 & 36.00 \\
Estudios superiores & & \\
\hline
\end{tabular}

International Journal of Developmental and Educational Psychology 


\section{ANÁLISIS PRELIMINAR DE LAS PROPIEDADES PSICOMÉTRICAS DE UN INSTRUMENTO PARA EVALUAR LAS ACTITUDES HACIA LA SEXUALIDAD DE LAS PERSONAS CON DISCAPACIDAD FÍSICA (ASEX-PDF)}

El grupo inicial de 554 participantes se dividió aleatoriamente en dos submuestras. La primera, que se utilizó para realizar el Análisis Factorial Exploratorio (AFE), estuvo formada por 293 sujetos (41.98\% hombres y $58.02 \%$ mujeres) de los cuales el 53.24\% tenían entre 20-35 años, el 31.40\% edades entre 36-51 años, el 15.02\% entre 52-66 años y el 0.34\% entre 67-92 años. Mientras que el segundo subgrupo que se utilizó para realizar el Análisis Factorial Confirmatorio (AFC) quedó constituido por 261 sujetos (40.61\% hombres y 59.39\% mujeres) con una distribución de edad en la que el 58.24\% tenían entre 20-35 años, el 23.75\% edades entre 36-51 años, el 16.48\% entre 52-66 años, y el 1.53\% entre 67-92 años. No se han encontrado diferencias estadísticamente significativas entre las submuestras por género, ${ }^{2}=-0.325, p=0.754$, ni por edad, ${ }^{2}=0.030, p=0.862$.

\section{Instrumentos} PDF)

Instrumento para evaluar las Actitudes hacia la Sexualidad de las Personas con Discapacidad Física (ASEX-

Este instrumento fue diseñado por la Dra. Mํㅡㄹ Dolores Gil-Llario y un grupo de colaboradores del equipo SALUSEX. Basándose en la escala de actitudes respecto a la sexualidad de personas con discapacidad mental de Oliva y Calderón (2010), se reformularon algunos ítems adaptándolos a las características de las PDF y se incluyeron otros reactivos con sustento en la literatura. Este instrumento fue aplicado en diversos trabajos de investigación (Cano-Ortega y Gil-Llario, 2016; Díaz-Rodríguez y Gil-Llario, 2017), en los que mostró una consistencia interna que oscilaba entre 0.75 y 0.78 .

La versión inicial de esta escala está formada por 15 ítems que evalúan las actitudes que se poseen respecto a diferentes aspectos de la sexualidad de las PDF: intimidad, prácticas sexuales, interés sexual, etc. Las respuestas tienen un formato tipo Likert con 5 opciones que permiten indicar el nivel de acuerdo/desacuerdo con cada enunciado: $1=$ totalmente en desacuerdo, $2=$ bastante en desacuerdo, $3=$ ni de acuerdo ni en desacuerdo, $4=$ bastante de acuerdo, 5= totalmente de acuerdo. Seis de los reactivos tienen una redacción negativa (ítems 1, 2, 8, 10, 11 y 13), mientras que el resto están formulados positivamente.

Assessment of Attitudes Towards Sexuality of Individuals with Intellectual Disability (ASEXID; Gil-Llario et al., 2021)

Dicha escala se compone de 18 elementos que evalúan las actitudes que se poseen hacia diferentes aspectos relacionados con la sexualidad de PDI: intimidad, interés sexual, capacidad de control de impulsos, etc. Los ítems se distribuyen en tres subescalas: "Actitud normalizadora" (NOR-A) formado por 7 ítems que exponen una conceptualización positiva de la sexualidad de las PDI, equiparándola a la de las personas sin DI; "Actitud negadora" (NEG-A) compuesto por 5 ítems que reflejan la creencia de que existe un menor interés por la sexualidad en las PDI; y "Actitud paternalista" (PAT-A) formada por 6 elementos que expresan la preocupación que se deriva de la supuesta falta de control de los impulsos sexuales de las PDI. Las respuestas se puntúan en una escala tipo Likert con cinco opciones que indican su nivel de acuerdo/desacuerdo con cada enunciado (1 "totalmente en desacuerdo" a 5 "totalmente de acuerdo"). Respecto a su consistencia interna, todas las subescalas obtuvieron un índice de fiabilidad superior a 0.65 (NOR-A, = 0.86; NEG-A, =0.71 y PAT-A, =0.66).

\section{Procedimiento}

Para la construcción del ASEX-PDF, primeramente, se realizó una exhaustiva revisión bibliográfica para conocer la tendencia actitudinal de la sociedad hacia la sexualidad de las PDF, detectando los aspectos más controvertidos, y para indagar las escalas existentes focalizadas en la evaluación de este aspecto actitudinal. Seguidamente, se procedió al análisis de la escala de actitudes respecto a la sexualidad de personas con discapacidad mental de Oliva y Calderón (2010), se reformularon 4 de sus ítems y se redactaron 11 nuevos elementos. Durante este proceso, con el objetivo de evitar la aquiescencia, se tuvo en cuenta la inclusión tanto de ítems de formulación directa como ítems inversos. Al mismo tiempo, se amplió la escala de respuesta a cinco puntos para permitir respuestas neutras, mejorando la confiabilidad de este modo. 
Tras realizar estos pasos, se presentó un conjunto preliminar de ítems a un consejo asesor compuesto por 5 profesionales expertos en el campo de la discapacidad y de la salud sexual, que evaluaron la comprensión de los ítems sugiriendo cambios siempre que la redacción no resultaba lo suficientemente clara. Tras esta primera revisión teórica, se procedió a realizar una aplicación piloto de dicho instrumento administrándoselo a 10 profesionales de un centro ocupacional de la Comunitat Valenciana. Esto permitió que se realizaran nuevas revisiones respecto a la formulación de los ítems, mejorando su claridad y adoptándolos a un lenguaje más inclusivo.

En segundo lugar, para reunir la muestra se recurrió a datos extraídos en diferentes estudios de investigación enmarcados dentro de un proyecto financiado por el "Programa Estatal de I+D+i Orientado a los Retos de la Sociedad del Ministerio de Ciencia. Innovación y Universidades (Proyecto RTI2018-095538-B-100)", tal y como se detalla a continuación:

La muestra relativa a la población general fue obtenida mediante la difusión del enlace de la batería de instrumentos online por las redes sociales oficiales del grupo de investigación (página web, Facebook, Twitter, etc.) y contactando vía email con diversas asociaciones españolas a las que se les informaba sobre el estudio solicitándoles su colaboración. El único requisito para participar fue poseer un buen dominio del español, ser mayor de edad y dar su consentimiento informado.

La muestra relativa a profesionales que trabajaban con PDF, por su parte, había sido reclutada por medio de muestreo incidental no aleatorio en diferentes centros ocupacionales. El contacto se produjo a través de los directores/as de dichos centros a los que se les proporcionó información sobre el estudio, siendo a su vez beneficiarios sus usuarios del programa de educación afectivo-sexual SALUDIVERSEX (Gil-Llario et al., 2019). Los centros ocupacionales que accedieron a colaborar fueron los encargados de difundir los cuestionarios entre los profesionales que trabajan en el mismo centro. Los criterios de inclusión eran tener contacto cercano con una PDF y poseer un buen dominio del español.

\section{Análisis de datos}

En primer lugar, se exploró la estructura factorial del ASEX-PDF mediante un AFE realizado con el software estadístico Factor 10.8.02. La extracción de factores se realizó a través de mínimos cuadrados ponderados diagonalmente y se aplicó una rotación oblicua (Normalized Oblimin Direct). Seguidamente, se comprobó el ajuste del modelo resultante a través de un AFC realizado con el software IBM SPSS Amos 24. Se aportan juntamente con el valor de Chi-cuadrado ( ${ }^{2}$ ), la información del Índice de Ajuste Comparativo (CFI), Incremental (IFI), Normado (NFI) y Tucker-Lewis (TLI). Los criterios de la literatura científica indican un ajuste del modelo satisfactorio con un CFI, IFI, TLI y NFI $\geq 0.95$ (Hu y Bentler, 1999). Sin embargo, también se consideraron los criterios menos estrictos de un buen ajuste (CFI, IFI, NFI y TLI $\geq 0.90$ ) (Marsh, Hau y Wen, 2004). Sobre el modelo factorial resultante, se extrajeron diversos estadísticos descriptivos de los ítems (rango, media, desviación típica, asimetría y curtosis), se exploraron las posibles diferencias significativas entre las actitudes presentadas respecto al género y al grupo de pertenencia de los participantes (t de Student), y se calculó el índice de fiabilidad del modelo resultante ( de Cronbach, ordinal y correlación ítem-test). También se obtuvo la validez convergente en términos del coeficiente de correlación de Pearson entre la puntuación general del ASEX-PDF y otras escalas empleadas para la medición de constructos semejantes.

\section{RESULTADOS}

\section{Análisis Factorial Exploratorio (AFE)}

Para comprobar la viabilidad de la aplicación de un AFE al instrumento ASEX-PDF, se calculó, en primer lugar, el índice de Kaiser-Meyer-Olkin (KM0 $=0.815)$ y la prueba de esfericidad de Bartlett ( ${ }^{2} 293=2106.9, p<$ .001), obteniendo resultados que indicaban que el cuestionario admitía una solución factorial. En consecuencia, se realizó un análisis paralelo de Horn de la matriz de correlaciones policórica/tetracórica, utilizando el estimador de mínimos cuadrados ponderados diagonalmente (DWLS) y rotación oblimin direct. Los resultados mostraron 
que un modelo unifactorial presentaba mejor ajuste de los datos (Tabla 2) que el modelo bifactorial, ya que este último reveló índices de ajuste más bajos.

Tabla 2 Matriz de componentes rotados, comunalidades (h2), valor propio y varianza explicada.

\begin{tabular}{|c|c|c|}
\hline & $\begin{array}{l}\text { Factor } \\
\text { I }\end{array}$ & $h^{2}$ \\
\hline $\begin{array}{l}\text { 1. Las personas con DF tienen menos interés por la sexualidad que las } \\
\text { personas sin ella }\end{array}$ & -0.17 & 0.03 \\
\hline $\begin{array}{l}\text { 2. Sólo se debe proporcionar educación sexual a las personas con DF } \\
\text { cuando éstas lo demandan }\end{array}$ & -0.06 & 0.00 \\
\hline 3. Me parece bien que las personas con DF se masturben ${ }^{a}$ & 0.87 & 0.75 \\
\hline $\begin{array}{l}\text { 4. Una persona con DF puede vivir su sexualidad como cualquier otra } \\
\text { persona }^{\text {a }}\end{array}$ & 0.64 & 0.41 \\
\hline 5. Las personas con DF deberían tener su espacio de intimidad ${ }^{\mathrm{a}}$ & 0.96 & 0.92 \\
\hline 6. Las personas con $\mathrm{DF}$ pueden tener pareja como las personas $\sin \mathrm{DF}^{\mathrm{a}}$ & 0.85 & 0.73 \\
\hline $\begin{array}{l}\text { 7. Me parece bien que las personas con DF se besen o acaricien con otra } \\
\text { persona }{ }^{a}\end{array}$ & 0.98 & 0.98 \\
\hline $\begin{array}{l}\text { 8. Me parece bien que las personas con DF tengan relaciones sexuales } \\
\text { siempre que no haya penetración }\end{array}$ & 0.20 & 0.04 \\
\hline $\begin{array}{l}\text { 9. Me parece bien que las personas con DF tengan relaciones sexuales } \\
\text { con o sin penetración }{ }^{\text {a }}\end{array}$ & 0.84 & 0.68 \\
\hline $\begin{array}{l}\text { 10. Las personas con DF necesitan que otro adulto tutor decida acerca } \\
\text { de su sexualidad }\end{array}$ & -0.19 & 0.03 \\
\hline 11. Las personas con DF son siempre heterosexuales & -0.25 & 0.06 \\
\hline 12. Me parece bien que las personas con DF vean pornografía ${ }^{a}$ & 0.63 & 0.40 \\
\hline $\begin{array}{l}\text { 13. Deberíamos evitar que las mujeres con DF se queden embarazadas } \\
\text { mediante el uso de anticonceptivos }\end{array}$ & 0.21 & 0.04 \\
\hline $\begin{array}{l}\text { 14. Me parece bien que las personas con DF contraten servicios } \\
\text { sexuales }\end{array}$ & 0.38 & 0.15 \\
\hline 15. Me parece bien que las personas con DF practiquen cibersexo ${ }^{a}$ & 0.62 & 0.38 \\
\hline Valor propio & 5.73 & \\
\hline$\%$ varianza explicada & 52.05 & \\
\hline
\end{tabular}

\footnotetext{
${ }^{a}$ Ítems que forman parte de la versión final del ASEX-PDF.
} 


\section{Análisis Factorial Confirmatorio (AFC)}

Para verificar la estructura, se realizó el AFC, usando el método robusto de máxima verosimilitud. El primer modelo factorial testado (M1) replicó exactamente la estructura factorial derivada del AFE, eliminando los ítems con saturaciones factoriales por debajo de .40 (ítems 1, 2, 8, 10, 11, 13 y 14). Los índices de ajuste obtenidos estaban por debajo de los valores de punto de corte: $\mathrm{CFI}=.734, \mathrm{IFI}=.735, \mathrm{NFI}=.730, \mathrm{TLI}=.609$.

Por esta razón, un segundo modelo (M2) fue testado incluyendo una serie de restricciones para mejorar su ajuste. Así, teniendo en cuenta los índices de modificación, se correlacionaron las covarianzas de los ítems 3, 9 y 12, ya que se trata de reactivos con una redacción similar (Long, 1983). Como se muestra en la Tabla 3, el valor de la 2 fue significativo $(p=.001)$, el CFI obtuvo un valor de 0.957 , semejante al IFI $(0.958)$ y al NFI (0.951), mientras que el valor del TLI fue menor (0.930). El modelo resultante puede verse en la Figura 1.

Tabla 3 Índices de bondad de ajuste para el AFC

\begin{tabular}{cccccccc}
\hline & $\chi^{2}$ & $\mathrm{gl}$ & $\mathrm{p}$ & CFI & IFI & NFI & TLI \\
\hline Modelo 1 & 686.86 & 19 & 0.001 & 0.734 & 0.735 & 0.730 & 0.609 \\
Modelo 2 & 124.29 & 17 & 0.001 & 0.957 & 0.958 & 0.951 & 0.930
\end{tabular}

Nota. $\chi^{2}$ Chi-cuadrado, $g l$ grados de libertad, p significancia del modelo general, CFI Índice de Ajuste Comparativo, IFI Índice de Ajuste Incremental, NFI Índice de Ajuste Normado, TLI Índice de Ajuste Tucker-Lewis

Fig. 1 Diagrama de ruta del modelo unifactorial

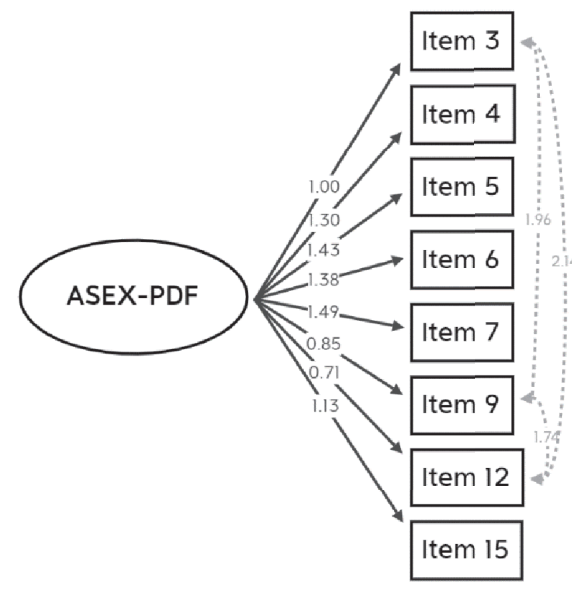

\section{Datos normativos y coeficientes de fiabilidad}

Como se aprecia en la Tabla 4, Ios ítems del ASEX-PDF presentan una distribución asimétrica negativa (Mo $\geq \mathrm{Me} \geq \mathrm{X}$ ), lo que significa que los valores más separados de la media se sitúan a la izquierda y que, por tanto, los ítems utilizados para explicar este constructo, en su mayoría, han sido respondidos de forma positiva, mostrando tolerancia. Al mismo tiempo, los ítems 5 ("Las personas con DF deberían tener su espacio de intimidad") y 7 ("Me parece bien que las personas con DF se besen o acaricien con otra persona") son los que presentan valores más altos de curtosis (Platikurtosis), por lo que se trataría de los reactivos más sensibles. Así mismo, 
estos también fueron los reactivos con una puntuación promedio más alta (ítem $5, M=4.80 ; D E=0.43$; ítem 7 , $M=4.83 ; D E=0.45$ ), mientras que la puntuación promedio más baja fue la del ítem 12 ("Me parece bien que las personas con DF vean pornografía"; $M=4.32, D E=1.10$ ) y 15 ("Me parece bien que las personas con DF practiquen cibersexo"; $M=4.31, D E=0.97$ ).

Por otro lado, la prueba t de Student no mostró diferencias de género estadísticamente significativas respecto a las actitudes $(F(1,554)=-0.84, p=0.40)$, aunque los hombres obtuvieron puntuaciones más bajas que las mujeres. En contra, sí se encontraron diferencias estadísticamente significativas entre los profesionales y la población general $(F(1,554)=158.82, p=0.000)$, siendo estos últimos quienes presentaban puntuaciones más bajas (Tabla 5).

Respecto a la consistencia interna del ASEX-PDF, el de Cronbach y el $\Omega$ ordinal obtuvieron valores por encima de 0.78 ( $=0.79, \Omega=0.89$ ). Las correlaciones ítem-test fueron excelentes con valores entre 0.56-0.79 (Tabla 4).

Tabla 4 Estadísticos descriptivos e índices de fiabilidad de los ítems y factores.

\begin{tabular}{ccccccccc}
\hline & \multicolumn{3}{c}{ Estadísticos descriptivos } & \multicolumn{3}{c}{ Índices de fiabilidad } \\
\hline Ítem & Rango & M & DE & Asimetría & Curtosis & $\alpha$ & $\Omega$ & I-T $r$ \\
\hline$(3)$ & $1-5$ & 4.63 & 0.77 & -2.70 & 7.00 & NA & NA & .79 \\
$(4)$ & $1-5$ & 4.49 & 0.89 & -2.15 & 4.22 & NA & NA & .56 \\
$(5)$ & $1-5$ & 4.80 & 0.43 & -4.66 & 22.29 & NA & NA & .69 \\
$(6)$ & $1-5$ & 4.73 & 0.57 & -3.36 & 11.25 & NA & NA & .61 \\
$(7)$ & $1-5$ & 4.83 & 0.45 & -4.64 & 21.44 & NA & NA & .65 \\
$(9)$ & $1-5$ & 4.68 & 0.67 & -2.87 & 7.95 & NA & NA & .74 \\
$(12)$ & $1-5$ & 4.32 & 1.10 & -1.52 & 1.50 & NA & NA & .73 \\
$(15)$ & $1-5$ & 4.31 & 0.97 & -1.33 & 1.06 & NA & NA & .61 \\
ASEX- & $8-40$ & 31.20 & 7.06 & -0.65 & 0.47 & .79 & .89 & NA \\
PDF & & & & & & & & \\
\hline
\end{tabular}

Tabla 5 Prueba $t$ de Student respecto al género y al grupo de pertenencia.

\begin{tabular}{|c|c|c|c|c|c|c|}
\hline & Hombre & Mujer & & $\begin{array}{c}\text { Profesion } \\
\text { ales }\end{array}$ & $\begin{array}{c}\text { Población } \\
\text { general }\end{array}$ & \\
\hline & $\mathrm{M}(\mathrm{DE})$ & $\mathrm{M}(\mathrm{DE})$ & $\mathrm{F}_{(1,554)}$ & $\mathrm{M}(\mathrm{DE})$ & $\mathrm{M}(\mathrm{DE})$ & $\mathrm{F}_{(1,554)}$ \\
\hline ASEX-PDF & $\begin{array}{l}30.90 \\
(6.87)\end{array}$ & $\begin{array}{l}31.41 \\
(7.19)\end{array}$ & -0.841 & $\begin{array}{l}38.05 \\
(1.58)\end{array}$ & $29.69(6.90)$ & $23.161 * * *$ \\
\hline
\end{tabular}

$* * * \mathrm{p}<0.001$ 


\section{Validez de criterio}

Para la estimación de la validez convergente de este instrumento, se calcularon los coeficientes de correlación de Pearson entre las tres subescalas del ASEXID y la puntuación total del instrumento ASEX-PDF. Como resultado de esto, se obtuvieron correlaciones positivas y estadísticamente significativas (a nivel $p<0.01$ ) entre el ASEX-PDF y las subescalas del ASEXID centradas en la evaluación de las actitudes normalizadas (NOR-A) y la preocupación (PAT-A) hacia la sexualidad de las PDI, mientras que la correlación fue negativa y estadísticamente significativa entre el ASEX-PDF y la subescala NEG-A centrada en la evaluación de creencias conservadoras acerca de la sexualidad de las PDI (Tabla 6).

Tabla 6 Correlaciones entre el ASEX-PDF y las subescalas del ASEXID.

\begin{tabular}{llll}
\hline & NOR-A & NEG-A & PAT-A \\
\hline ASEX-PDF & $0.672 * * *$ & $-0.228 * * *$ & $0.362 * * *$ \\
\hline$* * * \mathrm{p}<0.01$ & &
\end{tabular}

\section{DISCUSIÓN}

La sexualidad es una parte integral de la vida del ser humano que no siempre ha recibido la atención necesaria en el caso de las PDF. Actualmente, aunque las actitudes de la sociedad parecen estar cambiando, son pocas las investigaciones que exploran este cambio actitudinal debido, en parte, a la falta de instrumentos estandarizados centrados en la evaluación de las actitudes hacia la sexualidad de las PDF. Por ello, el objetivo del presente estudio ha sido la construcción y validación de un instrumento para evaluar las actitudes hacia la sexualidad de las PDF (ASEX-PDF) en el contexto español.

El ASEX-PDF es un instrumento autoadministrado de 8 ítems que fue diseñado para evaluar las actitudes de la sociedad hacia los diferentes aspectos involucrados en la sexualidad (prácticas sexuales, parejas sexuales, intimidad, material sexual online) de las PDF. En este sentido, este instrumento encaja con lo que se entiende por actitud sexual según López (2009) que la define como "predisposición a opinar, sentir y actuar ante objetos sexuales, situaciones, personas diferentes, normas o costumbres sociales y conductas sexuales", ya que los ítems de nuestro instrumento indagan en los diferentes aspectos que incluye dicho constructo: objetos sexuales (por ejemplo: Me parece bien que las personas con DF vean pornografía), situaciones (por ejemplo: Las personas con DF deberían tener su espacio de intimidad), personas diferentes (por ejemplo: Una persona con DF puede vivir su sexualidad como cualquier otra persona), normas o costumbres sociales (por ejemplo: Las personas con DF pueden tener pareja como las personas sin DF) y conductas sexuales (por ejemplo: Me parece bien que las personas con DFI se masturben).

De manera consistente con estudios previos que planteaban mayor aceptación de las relaciones sexuales en PDF por parte de las mujeres por su mayor predisposición al bienestar de los demás (Morales et al., 2011), los hombres de nuestra muestra han obtenido una puntuación media más baja que las mujeres, aunque estas diferencias no resultan significativas, al igual que sucedió en el estudio de Caballero-Gascón, et al. (2017) y de Sankhla y Theodore (2015), por lo que las actitudes hacia la sexualidad de las PDF no parecen diferir en función del género. En el caso de las comparaciones entre las actitudes hacia la sexualidad de las PDF de profesionales y población general, nuestros resultados manifiestan diferencias estadísticamente significativas, obteniendo los profesionales la puntuación total más elevada. Estos resultados van en la línea de lo revelado en el estudio de Chou, Lu y Lin (2018), y apoyan que se está produciendo un cambio de paradigma fruto de las formaciones sobre sexualidad que están recibiendo los profesionales en contacto con PDF.

Por otro lado, el análisis de sus propiedades psicométricas demostró que el modelo unidimensional es el que ofrece mejores índices de ajuste. Así mismo, todos los ítems muestran su adecuación, ya que las correlacio- 


\section{ANÁLISIS PRELIMINAR DE LAS PROPIEDADES PSICOMÉTRICAS DE UN INSTRUMENTTO PARA EVALUAR LAS ACTITUDES HACIA LA SEXUALIDAD DE LAS PERSONAS CON DISCAPACIDAD FÍSICA (ASEX-PDF)}

nes ítem-test obtuvieron valores por encima de 0.30 (Nunnally y Bernstein, 1995). Respecto a la consistencia interna, la escala presentó un de Cronbach de 0.89 , un valor mayor que el obtenido en los estudios de CanoOrtega y Gil-Llario (2016) y Díaz-Rodríguez y Gil-Llario (2017) con una muestra de características similares a la nuestra (familiares, profesionales y población general), y que otros estudios centrados en una muestra comunitaria (Palau-Cerisuelo y Salmerón-Sánchez, 2016). Estos resultados, junto con los valores del $\Omega$ ordinal, indican que el ASEX-PDF es un medida válida y fiable para evaluar las actitudes hacia la sexualidad de las PDF.

Este estudio también proporciona evidencia de validez convergente entre el ASEX-PDF y las diferentes subescalas del ASEXID. Los resultados son consistentes con los hallazgos de otras investigaciones, como la de Cano-Ortega y Gil-Llario (2016), que indicaban una relación positiva entre las actitudes hacia la sexualidad de las PDI y las actitudes hacia la sexualidad de las PDF. Así, a pesar de que la literatura solía reflejar actitudes más negativas hacia la sexualidad de las PDI que hacia las PDF (Hasson-Ohayon, et al., 2014), el estigma social y las barreras socioculturales han afectado y siguen afectando a ambos colectivos en el desarrollo y expresión de su sexualidad.

\section{CONCLUSIONES}

La falta de aceptación social de las necesidades emocionales y sexuales de las PDF limita las posibilidades de este colectivo de vivenciar de forma positiva su propia sexualidad. Dada esta realidad, se hace necesaria más investigación no solo dentro de la comunidad en general sino, sobre todo, entre aquellos grupos sociales en contacto directo con este colectivo, ya que la calidad, cantidad y tipo de información que reciban las PDF depende, en gran parte, de las actitudes de sus principales figuras de referencia.

La disponibilidad de un instrumento como el ASEX-PDF, que permita evaluar las actitudes hacia la sexualidad de las PDF, puede facilitar la investigación adicional de aspectos interrelacionados con la sexualidad, promover acciones que potencien el reconocimiento del derecho de las PDF a tener un desarrollo sexual saludable y, concienciarnos sobre la necesidad de formar a la sociedad y a los cuidadores directos de las PDF en la importancia de ofrecer una educación afectivo-sexual reglada y adaptada a las características del colectivo en cuestión.

Sin embargo, en futuras investigaciones deberían tenerse en cuenta que es posible que las actitudes difieran dependiendo de la limitación física que presente la PDF, por lo que sería importante que este aspecto fuera contemplado en la evaluación.

\section{REFERENCIAS BIBLIOGRÁFICAS}

Caballero-Gascón, L., Gil-Llario, M.D., Morell-Mengual, V., Fernández-García, O., y Gil-Juliá, B.: (2017). Actitudes de la población general hacia las relaciones sexuales de las Personas con diversidad funcional: variables moduladoras. Int. J. Dev. Educ. Psych., 4(1), 103-110. https://doi.org/10.17060/ijodaep.2017.n1.v4.1032

Cano-Ortega, L. y Gil-Llario, M.D. (2016). Actitudes hacia la sexualidad de las personas con discapacidad (Tesis de máster). Universitat de Valencia, Valencia.

Chou, Y.C., Lu, Z.Y.J., \& Lin C.J.: (2018). Comparison of attitudes to the sexual health of men and women with intellectual disability among parents, professionals, and university students. J. Intellect. Dev. Disabil. 43(2), 164-173. https://doi.org/10.3109/13668250.2016.1259465

Díaz-Rodríguez, I., y Gil-Llario, M.D. (2017). Construcción de la salud sexual y análisis de su influencia en la calidad de vida y prevención de abusos sexuales en personas adultas con discapacidad intelectual (Tesis doctoral). Universitat de Valencia, Valencia. https://roderic.uv.es/handle/10550/60078

East, L.J., \& Orchard, T.R. (2014). Somebody else's job: experiences of sex education among health professionals, parents and adolescents with physical disabilities in southwestern Ontario. Sex Disabil, 32, 335-50. https://doi.org/10.1007/s11195-013-9289-5

García Ortuño, V. (2017). Actitudes hacia la sexualidad de las personas con discapacidad: una propuesta de intervención (Tesis de grado). Universidad Miguel Hernández, Elche. 
Gil-Llario, M. D., Ballester-Arnal, R., Caballero-Gascón, L., y Escalera-Nieves, C. (2019). Programa SALUDIVERSEX. Programa de educación afectivo-sexual para adultos con diversidad funcional intelectual. Ediciones Pirámide.

Gil-Llario, M.D., Fernández-García, 0., Castro-Calvo, J., Caballero-Gascón L. \& Ballester-Arnal. R. (2021) Validation of a Tool to Assess Attitudes Towards Sexuality of Individuals with Intellectual Disability (ASEXID): A Preliminary Study. Sexuality and Disability, 39, 147-165. https://doi.org/10.1007/s11195-020-09650-3

Hasson-Ohayon, I., Hertz, I., Vilchinsky, N., \& Kravetz, S. (2014). Attitudes toward the sexuality of persons with physical versus psychiatric disabilities. Rehabilitation Psychology, 59(2), 236-241. https://doi.org/10.1037/a0035916

Hu, L.T. y Bentler, P.M. (1999). Cut-off criteria for fit indexes in covariance structure analysis: Conventional criteria versus new alternatives. Struct. Equ. Modeling. 6(1), 1-55 https://doi.org/10.1080/10705519909540118

Jemtå L, Fugl-Meyer KS, \& Öberg K. (2008) On intimacy, sexual activities and exposure to sexual abuse among children and adolescents with mobility impairment. Acta Paediatr, 97, 641-6. https://doi.org/10.1111/j.1651-2227.2008.00757.x

Kravetz, S., Katz, S., \& Albez, D. (1994). Attitudes toward Israeli war veterans with disabilities: Combat vs. noncombat military service and responsibility for the disability. Rehabilitation Counseling Bulletin, 37, 371-379.

Long, J. S. (1983). Confirmatory factor analysis. Beverly Hills (CA): Sage.

López, F. (2009). La educación sexual de los hijos. Pirámide, Madrid

Marsh, H.W., Hau, K.T. y Wen, Z. (2004). In Search of Golden Rules: Comment on Hypothesis-Testing Approaches to Setting Cutoff Values for Fit Indexes and Dangers in Overgeneralizing Hu and Bentler's (1999) Findings. Struct. Equ. Modeling. 11(3), 320-341. https://doi.org/10.1207/s15328007sem1103_2

Martínez-Ríos, B. (2013). Pobreza, discapacidad y derechos humanos. Revista Española de Discapacidad. 1(1), 9-32. http://dx.doi.org/10.5569/2340-5104.01.01.01

Morales, G., López, E., Charles, D., Tuero, Z., y Mullet, E. (2011). Actitudes hacia la sexualidad de las personas con discapacidad intelectual. Ciencia UANL., 14(4), 436-444

Morell-Mengual, V., Gil-Llario, M.D., Díaz-Rodríguez, I., y Caballero-Gascón, L.: (2017). Actitudes de padres, profesionales y población general hacia la sexualidad de las personas con discapacidad física e intelectual. Int. J. Dev. Educ. Psych., 4(1), 173-183. https://doi.org/10.17060/ijodaep.2017.n1.v4.1040

Nunnally, J. C., y Bernstein, I. J. (1995). Teoría psicométrica. McGrawHill.

Oliva, L. y Calderón, M. (2010). Elaboración de una escala de actitudes respecto a la sexualidad de personas con discapacidad mental. Revista Electrónica de Psicología Iztacala, 13(4), 106-115.

Palau-Cerisuelo, S. y Salmerón-Sánchez, P. (2016). Actitudes hacia la sexualidad de las personas con discapacidad (Tesis de máster). Universitat Jaume I, Castellón de la Plana. http://repositori.uji.es/xmlui/bitstream/handle/10234/165255/TFM_2015_palauS.pdf?seq\#: :text=En\%20e ste\%20estudio\%2C\%20se\%20considera,una\%20actitud\%20negativa\%20(0\%20conservadora

Ride, G., \& Newton, D. C. (2018). Exploring professionals' perceptions of the barriers and enablers to young people with physical disabilities accessing sexual and reproductive health services in Australia. Sexual health, 15(4), 312-317. https://doi.org/10.1071/SH17106

Salehi, M., Tavakol, H. K., Shabani, M., \& Ziaei, T. (2015). The relationship between self-esteem and sexual selfconcept in people with physical-motor disabilities. Iranian Red Crescent Medical Journal, 17(1). https://doi.org/10.5812/ircmj.25359

Sankhla, D., y Theodore, K. (2015). British attitudes towards sexuality in men and women with intellectual disabilities: a comparison between White Westerners and South Asians. Sex. Disabil., 33(4), 429-445. https://doi.org/10.1007/s11195-015-9423-7

Torices Rodarte, I. (2007) La sexualidad y la discapacidad física. Editorial MAD Eduforma. 


\section{ANÁLISIS PRELIMINAR DE LAS PROPIEDADES PSICOMÉTRICAS DE UN INSTRUMENTO PARA EVALUAR \\ LAS ACTITUDES HACIA LA SEXUALIDAD DE LAS PERSONAS CON DISCAPACIDAD FÍSICA (ASEX-PDF)}

World Health Organization and World Bank (2011). World report on disability. Geneva: WHO.

Zárate, 0., y Calderón, V. (2010). Elaboración de una escala de actitudes respecto a la sexualidad de personas con discapacidad mental. Revista Electrónica de Psicología Iztacala, 13(4), 106-115.

\section{NOTAS}

${ }^{1}$ Esta investigación ha sido realizada gracias a un proyecto de investigación financiado por el Programa Estatal de I+D+i de la Convocatoria 2018 Orientada a los Retos de la Sociedad del Ministerio de Ciencia. Innovación y Universidades (Proyecto RTI2018-095538-B-100 